\title{
Management and Prognosis of Early Postpartum Hemorrhage in African Low Setting Health
}

\section{Youssouf Traoré $1^{*}$, Ibrahima Téguété1, Amadou Bocoum¹, Mamadou Traoré1, Seydou Dao, Marion Koko Bomini2 ${ }^{2}$ Niani Mounkoro' ${ }^{1}$ Amadou Dolo ${ }^{1}$}

\author{
${ }^{1}$ Department of Gynecology and Obstetrics, Gabriel Touré Teaching Hospital, Bamako, Mali \\ ${ }^{2}$ Department of Gynecology and Obstetrics, Yopougon Teaching Hospital, Abidjan, Côte d'Ivoire \\ Email: ${ }^{\star}$ dryoussouf.traore@gmail.com
}

How to cite this paper: Traoré, Y., Téguété, I., Bocoum, A., Traoré, M., Dao, S., Bomini, M.K., Mounkoro, N. and Dolo, A. (2018) Management and Prognosis of Early Postpartum Hemorrhage in African Low Setting Health. Open Journal of Obstetrics and Gynecology, 8, 1-9. https://doi.org/10.4236/ojog.2018.81001

Received: November 15, 2017

Accepted: January 1, 2018

Published: January 4, 2018

Copyright ( 92018 by authors and Scientific Research Publishing Inc. This work is licensed under the Creative Commons Attribution International License (CC BY 4.0).

http://creativecommons.org/licenses/by/4.0/

\section{(c) () Open Access}

\begin{abstract}
Early postpartum hemorrhage is one of the major causes of maternal death in the world especially in developing countries. Its management often relieves resuscitation that is often difficult to set up in our countries and sometimes based on invasive and mutilate surgery. Objectives: The purpose of this survey was to report frequency of this pathology, to describe its management and the factors that influence the prognosis of early postpartum hemorrhage in low setting health in Africa. Method: Authors conducted a prospective study that analyzed early postpartum hemorrhage in the motherhoods of Gabriel Touré teaching hospital and community five health reference center of the district of Bamako. It took place from January, 2015 to December, 2016. The study concerned all the cases of early postpartum hemorrhage according to WHO definition. Statistical tests used were $\mathrm{X}^{2}$ or Fisher test, its $95 \%$ confidence interval $\left(\mathrm{CI}_{95 \%}\right)$, p value was significant if $<5 \%$. Results: Early postpartum hemorrhage frequency has been $0.7 \%$ ( 62 cases for 8.885 deliveries). Sixty nine and one percent (69.1\%) of patients have been blood fluid transfused. Obstetric treatment dominated by uterine revision (30.7\%). Hysterorraphy (4.0\%), hysterectomy (3.0\%), suture of uterus injuries (15.7\%), hypo gastric artery ligature (2.0\%) and B-Lynch compression suture (2.0\%) have been the main practiced surgical operations. No satisfy blood transfusion need was $26.9 \%$. The main risk factors of early PPH were high parity $(\mathrm{p}=0009 ; \mathrm{RR}=$ 3.04; $\left.\mathrm{CI}_{95 \%}[2.80-5.11]\right)$, prolonged labor $\left(\mathrm{p}=0004 ; \mathrm{RR}=4.00 ; \mathrm{CI}_{95 \%}[3.06-\right.$ $10.02])$, oxytocin/prostaglandin use $\left(\mathrm{p}=0003 ; \mathrm{RR}=1.47 ; \mathrm{CI}_{95 \%}[1.17-3.16]\right)$. Eleven of maternal occurred (11.8\%). Conclusion: Early postpartum hemorrhage is still a severe event in developing countries especially. Its management sometime consisted to invasive cares. Maternal prognosis that is influenced by unsatisfied blood need and late management is marked by high lethality.
\end{abstract}




\section{Keywords}

Early Postpartum Hemorrhage, Risk Factors, Surgery, Maternal Death

\section{Introduction}

Postpartum hemorrhage ( $\mathrm{PPH})$ is any excessive bleeding after delivery. It occurs in about $5 \%$ of all deliveries. Up to $25 \%$ of all pregnancy-related deaths are directly attributable to hemorrhage. Hemorrhage needs not be a sudden massive quantity; even steady moderate bleeding can lead to serious hypovolemia. It is the most common cause of maternal death worldwide [1] [2]. Globally, postpartum hemorrhage is a leading cause of maternal mortality [1]. The global prevalence of PPH is 6\% [2] and the highest burden is experienced in low-income countries [3] [4]. The magnitude of PPH in sub-Saharan Africa is high at 10.5\% [2]. In Mali, it constitutes the main cause of maternal mortality [3]. The trend is the same elsewhere in Africa.

Early postpartum hemorrhage (EPPH) remains despite important efforts undertaken by our African government is a crucial public health problem. If the maternal and neonatal mortality challenge has been taken up in advanced countries and in those where the health system is well organized, the countries in Sub-Saharan Africa have still not succeeded yet despite the increasing human, material and financial resources. In fact, in our countries, we can rarely forecast and prevent most of the early postpartum hemorrhage. With brutal beginning, it constitutes one of a major medico-surgical emergency happens suddenly.

Postpartum hemorrhage occurring is a dreadful complication for women. Its prognosis is sometimes grave for the mother.

One of the problems of operational definition of this disorder and even its treatment remains the estimation of maternal blood loss that must be done in real time in order to set up immediately an adapted treatment and avoid the infernal condition of the choc-hemostasis problems. The management of early postpartum hemorrhage must be coordinated and done as quickly as possible because any waste of time may have disastrous consequences. Its prevention is fewly efficient. It doesn't hinder early PPH to occur. This requires a better organization of our services in order to face early PPH when it occurs [4].

If in advanced countries, the treatment of early $\mathrm{PPH}$ is based on arterial embolization [5], and the vascular ligatures [6], in African countries, where there is no adequate technical equipment, the emphasis is put on of massage of the uterus, the suture of genital lesions and hysterectomy. The medical care is often difficult because patients are generally referred to hospital very late and in delicate conditions. This is the reason why the death rate is often high due to blood spoliation.

In order to better manage obstetrical emergencies, the government of Mali has implemented for more than thirty years the management policy of obstetrical 
and gynecologic emergencies based on the system of "reference and feedback" and for almost fifteen years the policy of the medical emergencies obstetrical and neonatal cares (EONC) has also been implemented. Through these policies, our country realizes a structural and organizational evolution of services (social and health agents' recruitment, service equipment, the implementation of the transfusion policy, the grant of free caesarian-section).

Gabriel Touré teaching hospital and the community five health reference center of the district are the most attended health structures and have largely taken profit of the resources allocated for this organization particularly the creation of small blood bank and equipment for emergency medical care. It is in this context that we have conducted this survey.

\section{Objectives}

The objectives were to report frequency of this pathology, to describe its management and the factors that influence the prognosis of early postpartum hemorrhage.

\section{Methods and Materials}

It is a descriptive and analytical study that has been carried out in the gynecology and obstetrical services of Gabriel Touré teaching hospital (level 3 of Malian health pyramid) and the community five health reference center of district of Bamako (level 2 of Malian health pyramid) from January 2015 to December 2016.

We have defined early postpartum hemorrhage as any hemorrhage occurring during the first 24 hours after delivery, and blood loss up than $500 \mathrm{ml}$ (after vaginal delivery) or up than $1000 \mathrm{ml}$ (after cesarean) or drawing to distort clinical situation of the patient [3].

Labor was considered prolonged in cases of prolonged latent phase and secondary arrest of cervical dilatation.

Including criteria: The study has accounted for the cases of hemorrhage that has been defined above.

Excluding criteria: women having suffered hemorrhage that does not correspond to the operating criteria defined above.

The data collection has been done through the delivery registers, delivery records and surgical report, death register.

The studied variables have been maternal epidemiologic characteristics, clinical factors that would influence the prognosis of the patient and the kind of treatment (medical, obstetrical and surgical).

The statistical tests used were the $\mathrm{X}^{2}$ and the Fisher test (according their application conditions), confident interval $95 \%\left(\mathrm{CI}_{95 \%}\right)$ and $\mathrm{p}$ value that was consider statistically significant if $<5 \%$.

This study has been approved by the ethics committee of the Faculty of Medicine, Pharmacy, and Dentistry at the University of Bamako, Mali. The pro- 
cedures of the management of all cases of postpartum hemorrhage have been performed according to the protocol and norms of reproductive health department of Mali.

\section{Results}

\subsection{Frequency}

We have recorded 93 cases of early postpartum hemorrhage on 8885 deliveries (1.04\%).

\subsection{Clinical and Biological Data}

The median age of patients was 29.2 years with an extreme from 15 to 45 years. They have been referred in emergency in $81.7 \%$ ( 76 cases). The median disparity was 6.1 with the extremes of 1 and 12 .

General state of patients was altered in almost $100 \%$ of cases $(95.7 \%)$ and characterized by a hemorrhagic shock.

The median rate of hemoglobin at the moment of admission was $7 \mathrm{~g} / \mathrm{dl}$ with extremes of 3 and $8 \mathrm{~g} / \mathrm{dl}$.

Forty five patients (48.4\%) didn't followed any prenatal consultation; Twenty women's pregnant had been at least one time followed by a health worker $(21.5 \%)$ and twenty eight pregnant women $(30.1 \%)$ had at least four followed up.

In Tables 1-3 we can see in multi various analyze risk factors influencing the occurrence of early postpartum hemorrhage. Calculation has been done on all the deliveries during the period of the study. Abrution placenta ( 35 cases), praevia placenta ( 5 cases) and uterus rupture (10 cases) were the three mains causes of antenatal hemorrhage.

\subsection{Prognosis}

Many risk factors were found in that survey as show in Table 4.

Five patients $(5.4 \%)$ died at the arrival before treatment starting. In 25 cases blood need was unsatisfied (26.9\%). Eleven patients died during or after management starting (11.8\%). The factors influencing maternal death were unsatisfied blood need ( $\mathrm{p}=0.000$; relative risk: $1.98 ; \mathrm{CI}_{95 \%}$ : $\left.[1.21-4.78]\right)$ and reference in emergency context of patients $\left(\mathrm{p}=0.001\right.$; relative risk: $4.55 ; \mathrm{CI}_{95 \%}$ : $[3.45-$ $12.00])$.

\section{Comments-Discussion}

\subsection{Limitation of This Survey}

The limitation of our survey is related to the fact that some patients who had serious medical conditions have been directly admitted in the intensive unit service in our hospital. That has probably leaded to bias of selection or information. Because of the type of this survey (retrospective data collection) some information in the files of patients were not available. 
Table 1. Multi various analyze of risk factors influencing the occurring of early postpartum hemorrhage in the two health centers concerning all the deliveries $(\mathrm{N}=8885)$.

\begin{tabular}{ccccc}
\hline Risk factors & Frequency of early Postpartum Hemorrhage & $\mathrm{p}$ & $\mathrm{RR}$ & $\mathrm{CI}_{95 \%}$ \\
\hline Antenatal hemorrhage $(\mathrm{n}=50)$ & 0.6 & 0.001 & 1.01 & {$[0.99-2.71]$} \\
Parity $>$ 5 $(\mathrm{n}=53)$ & 0.6 & 0.009 & 3.04 & {$[2.80-5.11]$} \\
Prolonged labor $(\mathrm{n}=71)$ & 0.8 & 0.004 & 4.00 & {$[3.06-10.02]$} \\
Oxytocin/prostaglandin used abuse $(\mathrm{n}=69)$ & 0.8 & 0.003 & 1.47 & {$[1.17-3.16]$} \\
\hline
\end{tabular}

Table 2. Causes of early postpartum hemorrhage in the two health structures.

\begin{tabular}{ccc}
\hline Causes of early PPH & Absolute frequency & Relative frequency (\%) \\
\hline Uterine atony & 38 & 40.9 \\
Retained placenta & 31 & 33.3 \\
Tissus injuries/laceration & 16 & 17.2 \\
Coagulation disorders & 8 & 8.6 \\
Total & 93 & 100.0 \\
\hline
\end{tabular}

Table 3. Surgical/obstetrical technics used for the management of early PPH in the two health centers $(\mathrm{n}=101)$.

\begin{tabular}{|c|c|c|}
\hline Management types & Absolute Frequency & Relative Frequency (\%) \\
\hline Manuel extraction of placenta & 40 & 39.6 \\
\hline Uterine revision & 31 & 30.7 \\
\hline Suture of lesions & 16 & 15.7 \\
\hline Hysterorraphy & 4 & 4.0 \\
\hline Hysterectomy & 3 & 3.0 \\
\hline Technic of Tsirulnikov & 3 & 3.0 \\
\hline Ligature of hypogastric artery & 2 & 2.0 \\
\hline B-Lynch compression suture & 2 & 2.0 \\
\hline
\end{tabular}

Some patients simultaneously received many types of technic for the management.

Table 4. Relation between maternal death and blood satisfaction need (BSN) for the management of postpartum hemorrhage in Gabriel Touré teaching hospital and the commune five's health center $(\mathrm{N}=93)$.

\begin{tabular}{|c|c|c|c|c|c|c|c|}
\hline \multirow{2}{*}{ Maternal death } & \multicolumn{2}{|c|}{ Satisfied need } & \multicolumn{2}{|c|}{ Non Satisfied Need } & \multirow{2}{*}{$\mathrm{P}$} & \multirow{2}{*}{$\mathrm{RR}$} & \multirow{2}{*}{$\mathrm{CI}_{95 \%}$} \\
\hline & Effective & $\%$ & Effective & $\%$ & & & \\
\hline No & 67 & 72.0 & 15 & 16.1 & 0.007 & 1.49 & {$[1.11-4.78]$} \\
\hline Yes & 1 & 1.1 & 10 & 10.8 & 0.000 & 9.81 & [5.40 - 12.86] \\
\hline
\end{tabular}

\subsection{Incidence, Risk Factors and Causes of Early Postpartum Hemorrhage}

The incidence of early PPH in our study is lower than the reported rates of Carroli et al. [7] (6\%), and Calvert et al. [8] (10.8\%) and whose found in Uganda [9] (9.0\%). This high incidence of PPH in this study may have been influenced by the characteristics of the study population; these were rural women that are reported to have higher rates of PPH [9]. Another reason that can explain the differences between data should be accounted for by the disparity of the operating 
definition of early postpartum hemorrhage that exists in different setting and including criteria. So, for some authors, a 10\% drop in hemoglobin or hematocrit is also used to define PPH. This does not always reflect the current status of the patient as bleeding continues. Also, the patient could seriously decompensate in the time it takes to get lab results. In our survey we only used WHO definition because of the difficulty to have in the context of emergency anytime hemoglobin or hematocrit result. Although incidences of IPPH are different in the studies above, this pathology is still frequent in developing countries [10] [11].

Although the prenatal consultation is important and consist an indispensable tool of prevention in obstetrics, it is sometimes limited concerning the prediction of early PPH. In fact, although more than half of the patients have done prenatal consultation, their obstetrical situation has been complicated of hemorrhage. This confirms thus the unpredictable and often unavoidable feature of this complication. That is the reason why it is necessary to provide services that offer the emergency obstetrical and neonatal cares. Therefore, hospitals providing obstetrical care should have in place $\mathrm{PPH}$ management protocols and a massive transfusion protocol [1].

We agree with authors who think while many risk factors are associated with $\mathrm{PPH}$, it often happens without warning [1].

The leading causes of IPPH known have been found in our survey and dominated by uterine atony (40.9\%) that is the most common cause as shown our data, retained placenta (33.3\%), lacerations (17.2\%) [12]. All that cases have some risk factors especially prolonged labor $\left(\mathrm{p}=0.004 ; \mathrm{RR}=4.00 ; \mathrm{CI}_{95 \%}[3.06-10.02]\right.$, use of oxytocin or prostaglandin for direction of labor or to induce labor $(\mathrm{p}=$ $003 ; \mathrm{RR}=1.47 ; \mathrm{CI}_{95 \%}[1.17-3.16]$, high parity of the patient. Literature review reveals others early PPH risk factors that were not found in our study. They are overdistended uterus related to multiple gestation, large baby or polyhydramnios, history of PPH, episiotomy, preeclampsia, operative delivery, chorioamnionitis.

\subsection{Management and Prognosis}

Physicians and nursing staff on labor and delivery should be skilled in accurately assessing blood loss and keenly aware that continued bleeding after delivery requires prompt evaluation and management. Quick recognition of early $\mathrm{PPH}$, along with identification of its cause and initiation of treatment, can avoid serious morbidity and mortality. Once PPH is diagnosed, the cause should be identified [1]. If elsewhere arterial embolization has been emphasized [5], in our service, we have focused as surgery management more on hemostasis hysterectomy (3.0\%). The realization of Tsirulnikov triple vascular ligation, the ligation of hypo gastric arterial and B-Lynch compression suture of uterine [13] [14] have permitted to stop the bleeding and to conserve the uterus in $7.0 \%$ of our patients. These technics should be best known by obstetricians in our maternity in order to ensure a better prognosis of patients who are victims of this complication. All the patients that early PPH was related to uterine atony received perfusion of oxytocin or/and prostaglandin according the WHO protocol. After 
excluded lacerations and retained placenta we used condom tamponade to temporize bleeding while awaiting the transfusion of blood products or transfer to surgical location [1] [15] [16] [17].

Treatment of IPPH involves volume replacement with crystalloid solutions and transfusion. That will permit to reduce risk of the occurrence of disseminated intravascular coagulopathy. Early postpartum hemorrhage has been at the origin of severe anemia in most of our patients, motivating thus blood transfusion. However, only $26.9 \%$ of need of transfusion has been satisfied. More than quarter of our patients has not received the number of packages of blood products that was necessary to care patients. The issue of blood transfusion is a challenge in Mali that any program has been able to take up. This accounts for current persistent difficulties concerning the constant availability of unstable blood products. There is only one blood bank which has the difficulty to cover the transfusion need of the district of Bamako. The policy of this blood bank is based on the donation of blood made by volunteer donors and on the substitution of the stock of blood whatever is the context (emergency or not) by individuals related to the family of the patient to transfuse the blood. In these conditions, the blood is often not available for patients received in emergency [3] causing the death somehow avoidable.

The risk of maternal death is highest in Sub-Saharan Africa [18] and the hemorrhage constitutes the main cause of this fatal outcome [19]. In a study evaluating the maternal mortality in Bamako, Teguete [3], reports overall rate superior to $10 \%$ of maternal death due to hemorrhage.

Institution of protocols for the treatment of postpartum hemorrhage can reduce mortality associated with hemorrhage. The maternal prognosis has been strongly marked by eleven maternal deaths (11.8\%). The late in the management of these patients as well as the lack of blood availability have contributed to fail prognosis of our patients.

\section{Conclusion}

The frequency of early postpartum hemorrhage is relatively weak in Mali. Its management is often difficult because of permanent non availability of unstable blood products. Its maternal prognosis is bad characterized by the high rate of maternal death. The improvement of the availability of emergency obstetric and neonatal care mainly availability of blood products, should permit improving of the prognosis of this pathology.

\section{References}

[1] Schneider, K. and Belfort, M.A. (2017) Postpartum Hemorrhage. The American Journal of Orthopedic, 46, 543-547.

[2] Mousa, H.A. and Walkinshaw, S. (2001) Major Postpartum Haemorrhage. Current Opinion in Obstetrics and Gynecology, 13, 595-603. https://doi.org/10.1097/00001703-200112000-00008 
[3] Téguété, I., Traoré, Y., Dénis, N., Mounkoro, N., Traoré, M. and Dolo, A. (2010) A 19-Year Retrospective Investigation of Maternal Mortality at Point National Hospital, Bamako, Mali. International Journal of Gynecology \& Obstetrics, 108, 194-198. https://doi.org/10.1016/j.ijgo.2009.09.026

[4] ACOG Practice Bulletin: Clinical Management Guidelines for Obstetricians and Gynecologists Number 76. (2015) Postpartum Hemorrhage. Obstetrics \& Gynecology, 108, 1039-1047.

[5] Pais, SO., Glickman, M., Schwartz, P., Pingoud, E. and Berkowitz, R. (1980) Embolization of Pelvic Arteries for Control of Post Partum Hemorrhage. Obstetrics \& Gynecology, 55, 754-758.

[6] Waters, E.G. (1952) Surgical Management of Postpartum Hemorrhage with Special Reference to Ligation of Uterine Arteries. American Journal of Obstetrics \& Gynecology, 64, 1143-1148. https://doi.org/10.1016/0002-9378(52)90377-3

[7] Carroli, G., Cuesta, C., Abalos, E. and Gulmezoglu, A.M. (2008) Epidemiology of Postpartum Haemorrhage: A Systematic Review. Best Practice \& Research Clinical Obstetrics \& Gynaecology, 22, 999-1012. https://doi.org/10.1016/j.bpobgyn.2008.08.004

[8] Calvert, C., Thomas, S.L., Ronsmans, C., Wagner, K.S., Adler, A.J. and Filippi, V. (2012) Identifying Regional Variation in the Prevalence of Postpartum Haemorrhage: A Systematic Review and Meta-Analysis. PLoS One, 7, 411-414. https://doi.org/10.1371/journal.pone.0041114

[9] Ononge, S., Mirembe, F., Wandabwa, J. and Campbell, O.M.R. (2016) Incidence and Risk Factors for Postpartum Hemorrhage in Uganda. Reproductive Health, 13, 2-7. https://doi.org/10.1186/s12978-016-0154-8

[10] AbouZahr, C. (2003) Global Burden of Maternal Death and Disability. British Medical Bulletin, 67, 1-11. https://doi.org/10.1093/bmb/ldg015

[11] Sheldon, W.R., Blum, J., Vogel, J.P., Souza, J.P., Gulmezoglu, A.M. and Winikoff, B. (2014) Maternal WHOMSo, Newborn Health Research N. Postpartum haemorrhage management, risks, and maternal outcomes: findings from the World Health Organization Multicountry Survey on Maternal and Newborn Health. BJOG, 121, 5-13. https://doi.org/10.1111/1471-0528.12636

[12] Traoré, Y., Mounkoro, N., Traoré, Dicko, F., et al. (2009) Uterus Rupture in Malian Rural Area. Annale de la SOGGO, 12, 11-15.

[13] Allams, M.S. and B-Lynch, C. (2005) The B-Lynch and Other Uterine Compression Suture Techniques. Int J Gynaecol Obstet, 89, 236-241.

https://doi.org/10.1016/j.ijgo.2005.02.014

[14] Cunningham, F.G., Leveno, K.J., Bloom, S.L., et al. (2010) Williams Obstetrics. 23rd Edition, McGraw-Hill, New York.

[15] Leduc, D., Denidas, V., Lalonde, A.B. and Ballerman, C. (2009) Active Management of the Third Stage of Labor: Prevention and Treatment of Postpartum Hemorrhage. Journal of Obstetrics and Gynaecology Canada, 31, 980-993. https://doi.org/10.1016/S1701-2163(16)34329-8

[16] Doumouchtsis, S.K., Papageorghiou, A.T. and Arulkumaran, S. (2007) Systematic Review of Conservative Management of Postpartum Hemorrhage: What to Do When Medical Treatment Fails. Obstetrical \& Gynecological Survey, 62, 540-547. https://doi.org/10.1097/01.ogx.0000271137.81361.93

[17] Dildy, G.A. (2002) Postpartum Hemorrhage: New Management Options. Clinical Obstetrics and Gynecology, 45, 330-344. https://doi.org/10.1097/00003081-200206000-00005 
[18] World Health Organization (2007) Maternal Mortality in 2005: Estimate Developed by WHO, UNICEF, UNFPA, and World Bank. WHO, Geneva.

[19] Khan, K.S., Wojdyla, D., Say, L., Gulmezoglu, A.M. and Van Look, P.F. (2006) WHO Analysis of Causes of Maternal Death: A Systematic Review. Lancet, 367, 1066-1074. https://doi.org/10.1016/S0140-6736(06)68397-9 